\title{
Phase Equilibria of Quasi-Ternary Systems Consisting of Multicomponent Polymers 1 and 2 in a Single Solvent I. Spinodal Curve and Critical Solution Point
}

\author{
Kenji Kamide, Shigenobu MAtsuda, and Hironobu SHIRATAKI \\ Fundamental Research Laboratory of Fibers and Fiber-Forming Polymers, \\ Asahi Chemical Industry Co., Ltd., 11-7, Hacchonawate, \\ Takatsuki, Osaka 569, Japan
}

(Received April 9, 1988)

\begin{abstract}
Theoretical equations of spinodal and neutral equilibrium conditions were derived, based on modified Flory-Huggins theory and without further approximation, for quasiternary solutions consisting of a multicomponent polymer 1 /multicomponent polymer $2 /$ single solvent. Numerous and systematic computer experiments clarified the effects of three thermodynamic interaction parameters between solvent-polymer $1\left(\chi_{01}\right)$, solvent-polymer $2\left(\chi_{02}\right)$, and polymer 1-polymer $2\left(\chi_{12}\right)$, the weight average degrees of polymerization of original polymers 1 and $2\left(X_{w}{ }^{0}\right.$ and $\left.Y_{w}{ }^{0}\right)$ and the breadth of the molecular weight distribution of original polymers $\left(X_{w}{ }^{0} / X_{n}{ }^{0}\right.$ and $Y_{w}{ }^{0} / Y_{n}{ }^{0} ; X_{n}{ }^{0}$ and $Y_{n}{ }^{0}$ are the number-a verage degree of polymerization, $X$ and $Y$, of the original polymers) on the spinodal curve and critical solution points. Here, the possible compositional dependence of three $\chi$ parameters were not considered.
\end{abstract}

KEY WORDS Multicomponent Polymers / Quasi-Ternary Polymer

Solution / $\chi$-Parameter / Average Degree of Polymerization / Spinodal

Curve / Critical Solution Point /

For the past several years studies have been actively performed on two liquid phase equilibria of quasi-ternary systems consisting of multicomponent polymer 1 , multicomponent polymer 2 and a single solvent. ${ }^{1-9} \operatorname{Scott}^{10}$ first studied theoretically the coexisting curve, spinodal curve (SC), and critical solution point (CSP) of the solution consisting of single component polymer 1 , single component polymer 2 , and a single low molecular solvent (rigorous ternary. system) to drive the approximate equations of CSP (eq 17a - c of ref 10 ). Note that these equations are only effective under the conditions of $\left|\chi_{01}-\chi_{02}\right|<1$ and $(X)^{1 / 2}<Y<$ $X^{2}$. Here, $\chi_{01}\left(\right.$ or $\chi_{02}$ ) is the thermodynamic interaction parameter between solvent and polymer 1 (or 2) and $X$ and $Y$, the degree of polymerization of polymers 1 and 2 , respectively. Soon after, Tompa ${ }^{11}$ succeeded in deriv- ing the rigorous equations giving SC and CSP for these ternary system. Spinodal and plait point conditions obtained by him are, ${ }^{11,12}$

$$
\begin{aligned}
& \sum x_{i} v_{i}-2 \sum x_{i} x_{j}\left(\chi_{i}-\chi_{j}\right) \\
& \quad+4 x_{0} x_{1} x_{2}\left(\chi_{0} \chi_{1}+\chi_{0} \chi_{1}+\chi_{1} \chi_{2}\right) v_{0} v_{1} v_{2}=0
\end{aligned}
$$

and

$$
\sum \frac{x_{i}^{2} v_{i}}{\left(1-2 \chi_{i} x_{i} v_{i}\right)^{3}}=0
$$

where

$$
\begin{aligned}
& \chi_{0}=\chi_{01}+\chi_{02}-\chi_{12} \\
& \chi_{1}=\chi_{01}+\chi_{12}-\chi_{12} \\
& \chi_{2}=\chi_{02}+\chi_{12}-\chi_{01}
\end{aligned}
$$

and $\chi_{12}$, the thermodynamic interaction parameter between polymers 1 and $2 ; v_{0}, v_{1}$, 
and $v_{2}$, the volume fractions of solvent, polymers 1 and $2 ; x_{0}=1, x_{1}=X$, and $x_{2}=Y$, respectively. Scott ${ }^{10}$ and Tompa ${ }^{11,12}$ calculated SC and CSP for a very specialized symmetrical case: $\chi_{01}=\chi_{02}$. Using the Tompa's equations (eq $1-3 \mathrm{c}$ ), Zemann and Patterson ${ }^{13}$ calculated SC and CSP for $\chi_{01} \neq \chi_{02}$, showing that a small difference in $\chi_{01}$ and $\chi_{02}$ has a marked effect on polymer compatibility. They also found that setting $\chi_{12}=0$ keeping $\chi_{01} \neq \chi_{02}$ leads to a closed loop region of incomplete miscibility ${ }^{13}$ although the binary mixture of polymer 1 and 2 shows complete miscibility (for example, see, Figures 1-4 of ref 13). Hsu and Prausnitz ${ }^{14}$ proposed a numerical procedure of calculating a coexisting curve (in other words, binodal curve; note that in this case, the binodal curve coincides with the cloud point curve (CPC) using Gibbs' phase equilibria condition. They examined in detail the effect of asymmetry of $\chi$ parameters between solvent and polymer 1 (or 2) (i.e., $\chi_{01} \neq \chi_{02}$ ) on the phase diagram and also examined the existence of close loop immissible region by putting $\chi_{12}$ lower than some critical value, ${ }^{14}$ semi-empirically determined. This critical value can be evaluated by eq 27 in this article. Recently, Solc ${ }^{15}$ proposed equations of CPC and CSP by introducing two new variables, $\eta$ and $\xi$, related to the two partition coefficients, $\sigma_{X}$ and $\sigma_{\mathrm{Y}}$, through $\eta^{2}=\sigma_{X} \sigma_{\mathrm{Y}}$ and $\xi^{2}=\sigma_{Y} / \sigma_{X}$. The overall polymer volume fraction $v\left(=v_{1}+v_{2}\right)$ at CSP $\left(v_{c}\right)$ was expressed, by use of CPC equation (eq 4 of ref 15) at the limit of $\sigma_{X}\left(\right.$ and $\left.\sigma_{Y}\right) \rightarrow 0$, in a simple closed form as follows; ${ }^{15}$

$$
\left[\frac{v_{c}}{1-v_{c}}\right]^{2}=x_{0} \frac{w_{1} x_{1}{ }^{2} \xi^{-3}+w_{2} x_{2}{ }^{2} \xi^{3}}{\left(w_{1} x_{1} \xi^{-1}+w_{2} x_{2} \xi^{3}\right.}
$$

where $w_{1}$ and $w_{2}$ are the volume compositions of polymers 1 and $2\left(w_{1}=v_{1} / v\right.$ and $\left.w_{2}=v_{2} / v\right)$. Šolc $^{16}$ also studied general conditions for multiple critical points using double roots of his CPC equation. All the above theoretical studies were limited to a monodisperse polymer 1 /monodipserse polymer 2 /single sol- vent system.

In 1970, a theoretical study on the phase equilibrium of multicomponent polymers 1 / multicomponent polymers $2 /$ single solvent system was first carried out by Koningsveld, Chermin, and Gordon. ${ }^{17,18}$ Note that they cited an equation of spinodal condition (eq 12 of ref 17) originally derived by Chermin (unpublished work) and they described an equation of neutral equilibrium condition (eq 13 of ref 17) without detailed derivation. As far as the authors know, Chermin's work still remains to be unpublished. Can we put our unconditional confidence in and employ for further calculations the equations derived without any mathematical detail? Koningsveld et al. showed experimental prism phase diagrams (i.e., $T-v_{1}-v_{2}$ space; $T$, Kelvin Temperature) of polyethylene/isotactic polystyrene/diphenylether ${ }^{17}$ and linear polyethylene/isotactic polypropylene/diphenylether systems, ${ }^{17,18}$ and deduced, from the experimental diagrams on quasi-ternary systems and by analogy of quasi-binary (multicomponent polymers/single solvent) systems, the form of interaction parameters $\left(\chi_{k l} ; k l=01,02\right.$, and 12) with the help of the spinodal equation (eq 27 of ref 17 ; rewritten form of eq 12 of ref 17) as ${ }^{17}$;

$$
\chi_{k l}=\chi_{k l, 1}+\frac{\chi_{k l, 2}}{T}+\chi_{k l, 3} T
$$

where $\chi_{k l, 1}, \chi_{k l, 2}$, and $\chi_{k l, 3}$ are the temperature independent coefficients $(k l=01,02$, and 12). However, they did not carry out systematic computer experiments based on their equations (eq 12 and 13 of ref 17) of spinodal and neutral equilibrium. The above situations attracted our attention and motivated a thermodynamical study on the SC and CSP as a further extension of Kamide and Matsuda's previous study ${ }^{19}$ of SC and CSP on systems consisting of a multicomponent polymer, solvent 1 and solvent 2 (see, eq 10 and 11 of ref 19).

In this article, we show some detailed derivation of themodynamical equations giving 
SC and the CSP and disclose the effect of the average molecular weight and molecular weight distribution of the two kinds of original polymers and of the three thermodynamical interaction $\chi$ parameters, $\chi_{01}, \chi_{02}$, and $\chi_{12}$ on SC and CSP.

\section{THEORETICAL BACKGROUND}

According to the Flory-Huggins' lattice theory of the dilute polymer solutions, Gibbs' free energy change of mixing $\Delta G$ is given by ${ }^{20}$

$$
\Delta G=R T L\left[v_{0} \ln v_{0}+\sum_{i=1}^{m_{1}} \frac{v_{X_{i}}}{X_{i}} \ln v_{X_{i}}+\sum_{j=1}^{m_{2}} \frac{v_{Y_{j}}}{Y_{j}} \ln v_{Y_{j}}+\chi_{01} v_{0} v_{1}+\chi_{02} v_{0} v_{2}+\chi_{12} v_{1} v_{2}\right]
$$

where $R$ is the gas constant, $T$, the Kelvin temperature, $L$, total number of lattice points $\left(\equiv N_{0} \sum_{i=1}^{m_{1}} X_{i} N_{X_{i}}+\sum_{j=1}^{m_{2}} Y_{j} N_{Y_{j}}: N_{0}, N_{X_{i}}\right.$ and $N_{Y_{j}}$; the number of solvents, $X_{i}$-mer and $Y_{j}$-mer, respectively), $v_{0}$, the volume fraction of solvent, $v_{X_{i}}$, the volume fraction of $X_{i}$-mer of polymer $1, v_{Y_{j}}$, the volume fraction of $Y_{j}$-mer of polymer $2, v_{1}$ and $v_{2}$, the toral volume fraction of polymer 1 and the polymer 2 $\left(v_{1}=\sum_{i=1}^{m_{1}} v_{X_{i}}\right.$ and $\left.v_{2}=\sum_{j=1}^{m_{2}} v_{Y_{j}}\right), m_{1}$, and $m_{2}$ are the total number of components of polymer 1 and 2 , respectively.

The chemical potentials of solvent, polymer 1 ( $X_{i}$-mer), and polymer 2 ( $Y_{j}$-mer), $\Delta \mu_{0}, \Delta \mu_{X_{i}}$, and $\Delta \mu_{Y_{j}}$, are directly derived from eq 6 . The results are:

$$
\begin{aligned}
\Delta \mu_{0}= & R T\left[\ln v_{0}+\left(1-\frac{1}{X_{n}}\right) v_{1}+\left(1-\frac{1}{Y_{n}}\right) v_{2}+\chi_{01} v_{1}\left(1-v_{0}\right)+\chi_{02} v_{2}\left(1-v_{0}\right)-\chi_{12} v_{1} v_{2}\right] \\
\Delta \mu_{X_{i}}= & R T\left[\ln v_{X_{i}}-\left(X_{i}-1\right)+X_{i}\left(1-\frac{1}{X_{n}}\right) v_{1}+X_{i}\left(1-\frac{1}{Y_{n}}\right) v_{2}\right. \\
& \left.+X_{i}\left\{\chi_{12} v_{2}\left(1-v_{1}\right)+\chi_{01} v_{0}\left(1-v_{1}\right)-\chi_{02} v_{0} v_{2}\right\}\right], \quad\left(i=1, \cdots, m_{1}\right) \\
\Delta \mu_{Y_{j}}= & R T\left[\ln v_{Y_{j}}-\left(Y_{j}-1\right)+Y_{j}\left(1-\frac{1}{X_{n}}\right) v_{1}+Y_{j}\left(1-\frac{1}{Y_{n}}\right) v_{2}\right. \\
& \left.+Y_{j}\left\{\chi_{12} v_{1}\left(1-v_{2}\right)+\chi_{02} v_{0}\left(1-v_{2}\right)-\chi_{01} v_{0} v_{1}\right\}\right] \quad\left(j=1, \cdots, m_{2}\right)
\end{aligned}
$$

Here, $X_{n}$ and $Y_{n}$ are the number-average of $X_{i}$ and $Y_{j}$. We assume that (a) $\chi_{01}, \chi_{02}$, and $\chi_{12}$ are independent of the concentration and molecular weight of polymers, (b) the molar volume of solvent and segment of polymers 1 and 2 are the same, (c) solvent, polymers 1 and 2 are volumetrically additive, and (d) the density of solvent is the same as that of polymers 1 and 2 . Note that eq 8 and 9 are strictly symmetrical with regard to an exchange of polymer 1 and polymer 2 .

Thermodynamical requirement against the spinodal curve is that the second order differential of Gibbs' free energy $\Delta G$ should always be zero on the curve and then the conditions of spinodal are given by the $\left(m_{1}+m_{2}\right) \times\left(m_{1}+m_{2}\right)$ determinant in the form: 


$$
\left|\Delta G^{\prime}\right|=\left|\begin{array}{cccccc}
\Delta \bar{G}_{X_{1} X_{1}}^{\prime} & \Delta \bar{G}_{X_{1} X_{2}}^{\prime} & \cdots \Delta \bar{G}_{X_{1} X_{m 1}}^{\prime} & \Delta \bar{G}_{X_{1} Y_{1}}^{\prime} & \Delta \bar{G}_{X_{1} Y_{2}}^{\prime} & \cdots \Delta \bar{G}_{X_{1} Y_{m 2}}^{\prime} \\
\Delta \bar{G}_{X_{2} X_{1}}^{\prime} & \Delta \bar{G}^{\prime}{ }_{X_{2} X_{2}} & \cdots \Delta \bar{G}_{X_{2} X_{m 1}}^{\prime} & \Delta \bar{G}_{X_{2} Y_{1}}^{\prime} & \Delta \bar{G}_{X_{2} Y_{2}}^{\prime} & \cdots \Delta \bar{G}_{X_{2} Y_{m 2}}^{\prime} \\
\vdots & \vdots & \vdots & \vdots & \vdots & \vdots \\
\Delta \bar{G}_{X_{m 1} X_{1}}^{\prime} & \Delta \bar{G}_{X_{m 1} X_{2}}^{\prime} & \cdots \Delta \bar{G}_{X_{m 1} X_{m 1}}^{\prime} & \Delta \bar{G}_{X_{m 1} Y_{1}}^{\prime} & \Delta \bar{G}_{X_{m 1} Y_{2}}^{\prime} & \cdots \Delta \bar{G}_{X_{m 1} Y_{m}}^{\prime} \\
\Delta \bar{G}_{Y_{1} X_{1}}^{\prime} & \Delta \bar{G}_{Y_{1} X_{2}}^{\prime} & \cdots \Delta \bar{G}_{Y_{1} X_{m 1}}^{\prime} & \Delta \bar{G}_{Y_{1} Y_{1}}^{\prime} & \Delta \bar{G}_{Y_{1} Y_{2}}^{\prime} & \cdots \Delta \bar{G}_{Y_{1} Y_{m 2}}^{\prime} \\
\Delta \bar{G}_{Y_{2} X_{1}}^{\prime} & \Delta \bar{G}_{Y_{2} X_{2}}^{\prime} & \cdots \Delta \bar{G}_{Y_{2} X_{m 1}}^{\prime} & \Delta \bar{G}_{Y_{2} Y_{1}}^{\prime} & \Delta \bar{G}_{Y_{2} Y_{2}}^{\prime} & \cdots \Delta \bar{G}_{Y_{2} Y_{m 2}}^{\prime} \\
\vdots & \vdots & \vdots & \vdots & \vdots & \vdots \\
\Delta \bar{G}_{Y_{m 2} X_{1}}^{\prime} & \Delta \bar{G}_{Y_{m 2} X_{2}}^{\prime} & \cdots \Delta \bar{G}_{Y_{m 2} X_{m 1}}^{\prime} & \Delta \bar{G}_{Y_{m 2} Y_{1}}^{\prime} & \Delta \bar{G}_{Y_{m 2} Y_{2}}^{\prime} & \cdots \Delta \bar{G}_{Y_{m 2} Y_{m 2}}^{\prime}
\end{array}\right|=0
$$

Here, $\Delta G^{\prime}$ is the Gibbs' free energy change of mixing per unit volume defined by

$$
\begin{aligned}
& \Delta G^{\prime}=v_{0}\left(\frac{\Delta \mu_{0}}{V_{0}}\right)+\sum_{i=1}^{m_{1}} v_{X_{i}}\left(\frac{\Delta \mu_{X_{i}}}{X_{i} V_{0}}\right)+\sum_{j=1}^{m_{2}} v_{Y_{j}}\left(\frac{\Delta \mu_{Y_{j}}}{Y_{j} V_{0}}\right) \\
& \Delta \bar{G}^{\prime}{ }_{k l}=\left(\frac{\partial^{2} \Delta G^{\prime}}{\partial v_{k} \partial v_{1}}\right)_{T, P, v_{n}} \quad\left(k, l=X_{1}, X_{2}, \cdots, X_{m_{1}}, Y_{1}, Y_{2}, \cdots, Y_{m_{2}} ; n \neq k, l\right)
\end{aligned}
$$

At CSP, in addition to eq 10, the following neutral equilibrium (eq 13) condition should be satisfied concurrently.

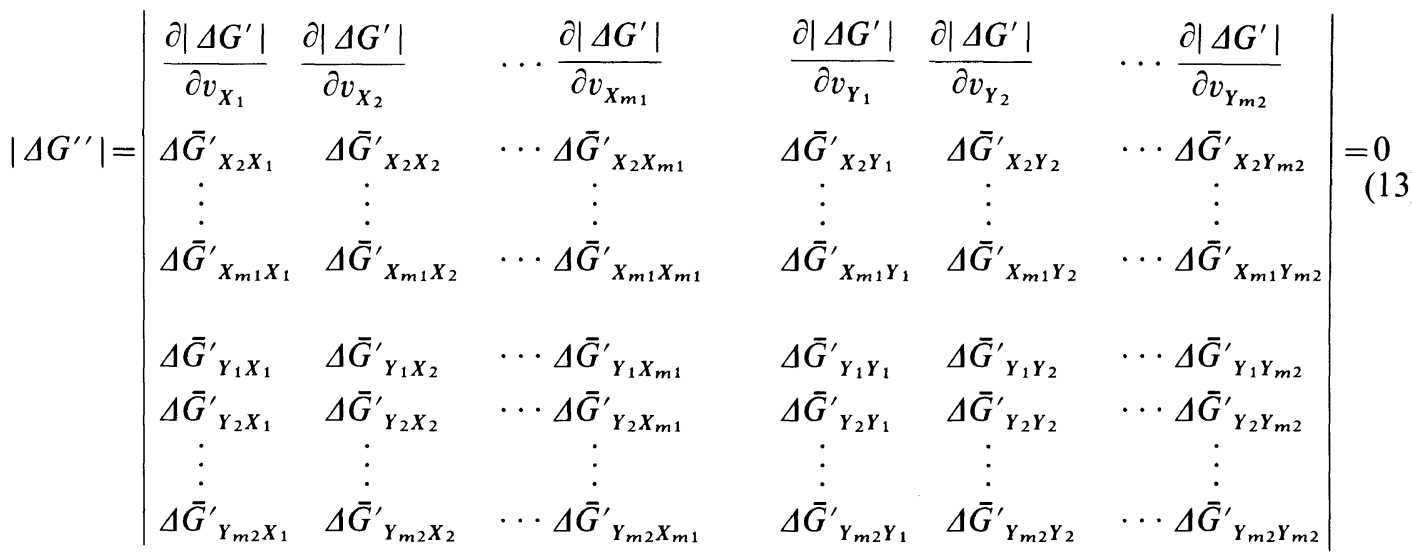

Gibbs' free energy change of mixing per unit volume $\Delta G^{\prime}$ can be rewritten by combination of eq 7-9 and 11 as:

$$
\Delta G^{\prime}=\left(\frac{R T}{V_{0}}\right)\left[v_{0} \ln v_{0}+\sum_{i=1}^{m_{1}} \frac{v_{X_{i}} \ln v_{X_{i}}}{X_{i}}+\sum_{j=1}^{m_{2}} \frac{v_{Y_{j}} \ln v_{Y_{j}}}{Y_{j}}+\chi_{01} v_{0} v_{1}+\chi_{02} v_{0} v_{2}+\chi_{12} v_{1} v_{2}\right]
$$

Substitution of eq 14 into eq 12 yields five types of $\Delta \bar{G}^{\prime}{ }_{k l}$ :

$$
\begin{array}{ll}
\left(\frac{V_{0}}{R T}\right) \Delta \bar{G}^{\prime}{ }_{k l}=\frac{1}{v_{0}}-2 \chi_{01} \equiv M & \left(\text { for } k \neq l,(k, l)=\left(X_{i}, X_{j}\right)\right) \\
\left(\frac{V_{0}}{R T}\right) \Delta \bar{G}_{k l}^{\prime}=\frac{1}{v_{0}}-2 \chi_{02} \equiv N & \left(\text { for } k \neq l,(k, l)=\left(Y_{i}, Y_{j}\right)\right)
\end{array}
$$




$$
\begin{array}{ll}
\left(\frac{V_{0}}{R T}\right) \Delta \bar{G}^{\prime}{ }_{k l}=\frac{1}{v_{0}}+\chi_{12}-\chi_{01}-\chi_{02} \equiv K & \left(\text { for } k \neq l,(k, l)=\left(X_{i}, Y_{j}\right) \text { or }\left(Y_{j}, X_{i}\right)\right) \\
\left(\frac{V_{0}}{R T}\right) \Delta \bar{G}^{\prime}{ }_{k l}=\frac{1}{v_{0}}-2 \chi_{01}+\frac{1}{X_{i} v_{X_{i}}} \equiv M+M_{i} \quad\left(\text { for } k=l=X_{i}\right) \\
\left(\frac{V_{0}}{R T}\right) \Delta \bar{G}^{\prime}{ }_{k l}=\frac{1}{v_{0}}-2 \chi_{02}+\frac{1}{Y_{j} v_{Y_{j}}} \equiv N+N_{j} \quad\left(\text { for } k=l=Y_{j}\right)
\end{array}
$$

Spinodal condition (eq 10) can be rewritten with the help of eq 14, 15a-e as eq 16 and after some tedious calculations, eq 16 can be simplified into eq 16' (see, Appendix A).

$$
\begin{aligned}
\left|\Delta G^{\prime}\right|= & \left(\frac{R T}{V_{0}}\right)^{m_{1}+m_{2}}\left|\begin{array}{cccccccc}
M+M_{1} & M & \ldots & M & K & K & \cdots & K \\
M & M+M_{2} & \ldots & M & K & K & \cdots & K \\
\vdots & \vdots & & \vdots & \vdots & \vdots & & \vdots \\
M & M & & M+M_{m_{1}} & K & K & \cdots & K \\
K & K & \ldots & K & N+N_{1} & N & \cdots & N \\
K & K & \ldots & K & N & N+N_{2} & \cdots & N \\
\vdots & \vdots & & \vdots & \vdots & \vdots & \\
K & K & \cdots & K & N & N & \cdots & N+N_{\mathrm{m}_{2}}
\end{array}\right| \\
= & \left(\frac{R T}{V_{0}}\right)^{m_{1}+m_{2}}\left(\prod_{i=1}^{m_{1}} M_{i}\right)\left(\prod_{j=1}^{m_{2}} N_{j}\right) \\
& \times\left[\left(1+M \sum_{i=1}^{m_{1}} \frac{1}{M_{i}}\right)\left(1+N \sum_{j=1}^{m_{2}} \frac{1}{N_{j}}\right)-K^{2}\left(\sum_{i=1}^{m_{1}} \frac{1}{M_{i}}\right)\left(\sum_{j=1}^{m_{2}} \frac{1}{N_{j}}\right)\right]=0
\end{aligned}
$$

Spinodal condition is then given by:

$$
\left(1+M \sum_{i=1}^{m_{1}} \frac{1}{M_{i}}\right)\left(1+N \sum_{j=1}^{m_{2}} \frac{1}{N_{j}}\right)-K^{2}\left(\sum_{i=1}^{m_{1}} \frac{1}{M_{i}}\right)\left(\sum_{j=1}^{m_{2}} \frac{1}{N_{j}}\right)=0
$$

The neutral equilibrium conditions eq 13 can be rewritten through use of eq $15 \mathrm{a}-\mathrm{e}$ as eq 18 , which can also be simplified into eq 18' (see, Appendix A);

$$
\left|\Delta G^{\prime \prime}\right|=\left(\frac{R T}{V_{0}}\right)^{m_{1}+m_{2}-1}\left|\begin{array}{cccccccc}
W_{X_{1}} & W_{X_{2}} & \cdots & W_{X_{m 1}} & W_{Y_{1}} & W_{Y_{2}} & \cdots & W_{Y_{m 2}} \\
M & M+M_{2} & \cdots & M & K & K & \cdots & K \\
\vdots & \vdots & & \vdots & \vdots & \vdots & & \vdots \\
M & M & & M+M_{m_{1}} & K & K & \cdots & K \\
K & K & \cdots & K & N+N_{1} & N & \cdots & N \\
K & K & \cdots & K & N & N+N_{2} & \cdots & N \\
\vdots & \vdots & & \vdots & \vdots & \vdots & & \vdots \\
K & K & \cdots & K & N & N & \cdots & N+N_{m_{2}}
\end{array}\right|
$$




$$
\begin{aligned}
= & \left(\frac{R T}{V_{0}}\right)^{m_{1}+m_{2}-1}\left(\prod_{i=1}^{m_{1}} M_{i}\right)\left(\prod_{j=1}^{m_{2}} N_{j}\right)\left(M_{1} \sum_{i=1}^{m_{1}} \frac{1}{M_{i}}\right)^{-1} \\
& \times\left[\left(\sum_{i=1}^{m_{1}} \frac{W_{X_{i}}}{M_{i}}\right)\left(1+N \sum_{j=1}^{m_{2}} \frac{1}{N_{j}}\right)-K\left(\sum_{i=1}^{m_{1}} \frac{1}{M_{i}}\right)\left(\sum_{j=1}^{m_{2}} \frac{W_{Y_{j}}}{N_{j}}\right)\right]=0
\end{aligned}
$$

where $W_{X_{i}}$ and $W_{Y_{j}}$ are

$$
\begin{aligned}
W_{X_{i}} \equiv\left(\frac{\partial\left|\Delta G^{\prime}\right|}{\partial v_{X_{i}}}\right)_{T, P, v_{k}} \\
\left(k \neq X_{i}, k=X_{1}, \cdots, X_{m_{1}}, Y_{1}, \cdots, Y_{m_{2}}\right) \\
W_{Y_{j}} \equiv\left(\frac{\partial\left|\Delta G^{\prime}\right|}{\partial v_{Y_{j}}}\right)_{T, P, v_{k}} \\
\left(k \neq Y_{j}, k=X_{1}, \cdots, X_{m_{1}}, Y_{1}, \cdots, Y_{m_{2}}\right)
\end{aligned}
$$

The neutral equilibrium condition is then given by:

$$
\begin{aligned}
&\left(\sum_{i=1}^{m_{1}} \frac{W_{X_{i}}}{M_{i}}\right)\left(1+N \sum_{j=1}^{m_{2}} \frac{1}{N_{j}}\right) \\
&-K\left(\sum_{i=1}^{m_{1}} \frac{1}{M_{i}}\right)\left(\sum_{j=1}^{m_{2}} \frac{W_{Y_{j}}}{N_{j}}\right)=0
\end{aligned}
$$

Equations 17 and 20 agree completely with the Koningsveld-Chermin-Gordon's equations (eq 12 and 13 of ref 17), respectively. The parameters in eqs 17 and 20 can be replaced with experimentally determined parameters such as $v_{0}, v_{1}, v_{2}, \chi_{01}, \chi_{02}, \chi_{12}$, the weight- and $z$-average $X_{i}$ (and $\left.Y_{j}\right), X_{w}{ }^{0}$ and $X_{z}{ }^{0}\left(Y_{w}{ }^{0}\right.$ and $\left.Y_{z}^{0}\right)$ and theoretical expressions finally obtained from eq 17 and 20 :

$$
\begin{gathered}
\left(\frac{1}{v_{0}}+\frac{1}{v_{1} X_{w}{ }^{0}}-2 \chi_{01}\right)\left(\frac{1}{v_{0}}+\frac{1}{v_{2} Y_{w}{ }^{0}}-2 \chi_{02}\right) \\
-\left(\frac{1}{v_{0}}+\chi_{12}-\chi_{01}-\chi_{02}\right)^{2}=0
\end{gathered}
$$

$$
\begin{gathered}
{\left[\left(\frac{1}{v_{0}^{2}}-\frac{X_{z}^{0}}{\left(v_{1} X_{w}{ }^{0}\right)^{2}}\right)\left(\frac{1}{v_{0}}+\frac{1}{v_{2} X_{w}{ }^{0}}-2 \chi_{02}\right)\right.} \\
+\frac{1}{v_{0}^{2}}\left(\frac{1}{v_{0}}+\frac{1}{v_{1} X_{w}{ }^{0}}-2 \chi_{01}\right) \\
\left.-\frac{2}{v_{0}^{2}}\left(\frac{1}{v_{0}}+\chi_{12}-\chi_{01}-\chi_{02}\right)\right] \\
\quad \times\left(\frac{1}{v_{0}}+\frac{1}{v_{2} Y_{w}{ }^{0}}-2 \chi_{02}\right) \\
-\left[\left(\frac{1}{v_{0}^{2}}-\frac{Y_{z}^{0}}{\left(v_{2} Y_{w}^{0}\right)^{2}}\right)\left(\frac{1}{v_{0}}+\frac{1}{v_{1} X_{w}{ }^{0}}-2 \chi_{01}\right)\right. \\
\quad+\frac{1}{v_{0}^{2}}\left(\frac{1}{v_{0}}+\frac{1}{v_{2} Y_{w}^{0}}-2 \chi_{02}\right) \\
\left.-\frac{2}{v_{0}^{2}}\left(\frac{1}{v_{0}}+\chi_{12}-\chi_{01}-\chi_{02}\right)\right] \\
\times\left(\frac{1}{v_{0}}+\chi_{12}-\chi_{01}-\chi_{02}\right)=0
\end{gathered}
$$

with

$$
v_{0}+v_{1}+v_{2}=1
$$

Equation 21 shows good coincidence with eq 17 of ref 17 (Konigsveld-Chermin-Gordon's equation). Equations 21 and 22 are evidently symmetrical with respect to the exchange of polymers 1 and 2 , respectively, which is the thermodynamical requirement. The symmetrical forms of eq 21 and 22 are (see, Appendix B):

and 


$$
\begin{aligned}
& \left(\frac{1}{X_{w}{ }^{0} v_{1}}+\chi_{02}-\chi_{01}-\chi_{02}\right)\left(\frac{1}{Y_{w}{ }^{0} v_{2}}+\chi_{01}-\chi_{02}-\chi_{12}\right) \\
& \quad+\left(\frac{1}{v_{0}}+\chi_{12}-\chi_{01}-\chi_{02}\right)\left(\frac{1}{X_{w}{ }^{0} v_{1}}+\chi_{02}-\chi_{01}-\chi_{12}\right) \\
& \quad+\left(\frac{1}{v_{0}}+\chi_{12}-\chi_{01}-\chi_{02}\right)\left(\frac{1}{Y_{w}^{0} v_{2}}+\chi_{01}-\chi_{02}-\chi_{12}\right)=0
\end{aligned}
$$

and

$$
\begin{aligned}
& \frac{1}{v_{0}^{2}}\left(\frac{1}{X_{w}^{0} v_{1}}+\chi_{02}-\chi_{01}-\chi_{12}\right)^{3}\left(\frac{1}{Y_{w}{ }^{0} v_{2}}+\chi_{01}-\chi_{02}-\chi_{12}\right)^{3} \\
& +\frac{X_{z}^{0}}{\left(X_{w}^{0} v_{1}\right)^{2}}\left(\frac{1}{Y_{w}^{0} v_{2}}+\chi_{01}-\chi_{02}-\chi_{12}\right)^{3}\left(\frac{1}{v_{0}}+\chi_{12}-\chi_{01}-\chi_{02}\right)^{3} \\
& +\frac{Y_{z}^{0}}{\left(Y_{w}^{0} v_{2}\right)^{2}}\left(\frac{1}{X_{w}^{0} v_{1}}+\chi_{02}-\chi_{01}-\chi_{12}\right)^{3}\left(\frac{1}{v_{0}}+\chi_{12}-\chi_{01}-\chi_{02}\right)^{3}=0
\end{aligned}
$$

Then, SC can be calculated from eq 21 and $v_{1}$, $v_{2}$, and $v_{0}$ at CSP (referred to as $v_{1}{ }^{c}, v_{2}{ }^{c}$, and $v_{0}{ }^{c}$ ) can be evaluated by solving simultaneous equations (eq $21-23$ ).

For single component polymer $1 /$ single component polymer $2 /$ single solvent system, eq 21 and 22 reduce to those whose $X_{w}{ }^{0}$ (and $\left.X_{z}^{0}\right)$ and $Y_{w}{ }^{0}$ (and $Y_{z}^{0}$ ) are replaced by $X$ and $Y$, respectively.

$$
\left(\frac{1}{v_{0}}+\frac{1}{v_{X} X}-2 \chi_{01}\right)\left(\frac{1}{v_{0}}+\frac{1}{v_{Y} Y}-2 \chi_{02}\right)-\left(\frac{1}{v_{0}}+\chi_{12}-\chi_{01}-\chi_{02}\right)^{2}=0
$$

and

$$
\begin{aligned}
& {\left[\left(\frac{1}{v_{0}^{2}}-\frac{1}{v_{X}^{2} X}\right)\left(\frac{1}{v_{0}}+\frac{1}{v_{Y} X}-2 \chi_{02}\right)+\frac{1}{v_{0}^{2}}\left(\frac{1}{v_{0}}+\frac{1}{v_{X} X}-2 \chi_{01}\right)\right.} \\
& \left.-\frac{2}{v_{0}^{2}}\left(\frac{1}{v_{0}}+\chi_{12}-\chi_{01}-\chi_{02}\right)\right]\left(\frac{1}{v_{0}}+\frac{1}{v_{Y} Y}-2 \chi_{02}\right) \\
& +\left[\left(\frac{1}{v_{0}^{2}}-\frac{1}{v_{Y}^{2} Y}\right)\left(\frac{1}{v_{0}}+\frac{1}{v_{X} X}-2 \chi_{01}\right)+\frac{1}{v_{0}^{2}}\left(\frac{1}{v_{0}}+\frac{1}{v_{Y} Y}-2 \chi_{02}\right)\right. \\
& \left.-\frac{2}{v_{0}^{2}}\left(\frac{1}{v_{0}}+\chi_{12}-\chi_{01}-\chi_{02}\right)\right]\left(\frac{1}{v_{0}}+\chi_{12}-\chi_{01}-\chi_{02}\right)=0
\end{aligned}
$$

These equations agree exactly with those (eq 1 and 2) derived by Tompa. ${ }^{11,12}$ Inspection of eq 21 indicates that spinodal point is a function of $X_{w^{1}}{ }^{0}, Y_{w^{*}}{ }^{0}, \chi_{01}, \chi_{02}$, and $\chi_{12}$ and is independent of the polydispersity of the two polymers.

\section{COMPUTER EXPERIMENT}

Put left-hand side of eq 21 and 22 with $A$ and B. A and B (spinodal and neutral equilibrium equations) become the functions of two variables $v_{1}$ and $v_{2}$ by use of eq 23 when $\chi_{01}, \chi_{02}$, 
$\chi_{12}, X_{w}{ }^{0}, X_{z}{ }^{0}, Y_{w}{ }^{0}$, and $Y_{z}{ }^{0}$ are known as prerequisites. For given $v_{2}$, the spinodal equation can be represented by a single variable $v_{1}\left(A=A\left(v_{1}\right)=0\right)$ and $v_{1}$ at spinodal point can be evaluated using a single variable Newton's method. By solving two-variable $\left(v_{1}\right.$ and $v_{2}$ ) simultaneous eq 21 and 22 with the aid of two-variable Newton's method, CSP (namely $v_{1}{ }^{c}$ and $v_{2}{ }^{c}$ (and $\left.v_{0}{ }^{c}\right)$ ) can be evaluated.

The computer experiments of SC and CSP were performed under the following conditions: $\chi_{12}=-0.4 \sim 0.2, \chi_{01}$ and $\chi_{02}=0 \sim 2.0$, $X_{w}{ }^{0}$ and $Y_{w}{ }^{0}=1 \cdot 10^{6}$, and $X_{w}{ }^{0} / X_{n}{ }^{0}$ and $Y_{w}{ }^{0} /$ $Y_{n}^{0}=1 \sim 5$. The original polymer was assumed to have the Schulz-Zimm type molecular weight distribution (MWD). Note that $X_{z}{ }^{0}-X_{w}{ }^{0}=X_{w}{ }^{0}-X_{n}{ }^{0}$ and $Y_{z}{ }^{0}-Y_{w}{ }^{0}=Y_{w}{ }^{0}-$ $Y_{n}^{0}$ hold in this case. The calculations were performed with a FACOM M360.

\section{RESULTS AND DISCUSSION}

Figure 1 shows the effect of the polydispersity of polymers 1 and 2 on SC and CSP of the quasi-ternary solutions. Here the two polymers have the Schulz-Zimm distribution with $X_{w}{ }^{0}$ or $Y_{w}{ }^{0}=300$, and $\chi_{01}=0.2, \chi_{02}=0.3$, and $\chi_{12}=0.01$ are assumed. All CSPs are just on the spinodals as theory predicts, moving depending on polymer polydispersity. For a fixed $Y_{w}{ }^{0} / Y_{n}{ }^{0}(=2)$ CSP shifts to the side of polymer 1 as $X_{w}{ }^{0} / X_{n}^{0}$ increases from 1 to 5 . Reversely, at a fixed $X_{w}{ }^{0} / X_{n}{ }^{0}(=2)$ CSP shifts to the side of polymer 2 as $Y_{w}{ }^{0} / Y_{n}{ }^{0}$ incerases. Namely, CSP moves to the side of the polymer whose MWD is widened. This coincides well with the relation between CSP and the polydispersity of multicomponent polymer dissolved in single solvent or of multicomponent polymer in binary solvent mixture systems. ${ }^{19}$ Evidently, the CSPs for the multicomponent polymer deviate significantly from that for a single component polymer, at least in the range $X_{u^{1}}{ }^{0} / X_{n}{ }^{0}$ or $Y_{w}{ }^{0} / Y_{n}{ }^{0} \gtrsim 1.05$. The CSP locates at peak of the SC when the polymolecularities of both polymers coincide with each other (in
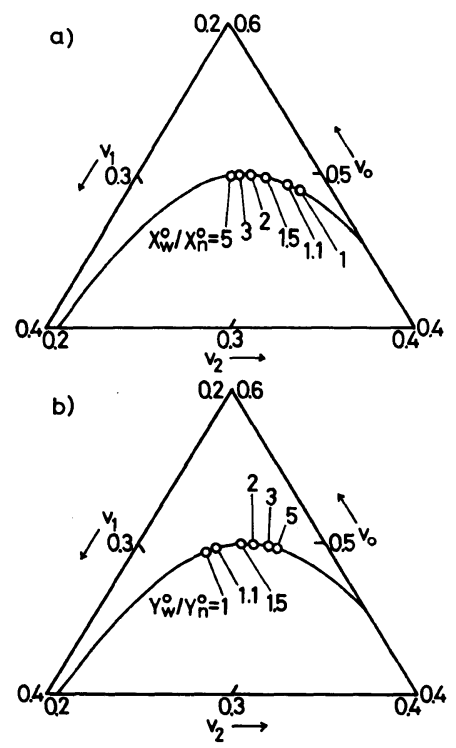

Figure 1. Effects of $X_{w}^{0} / X_{n}^{0}$ (a) and $Y_{w}{ }^{0} / Y_{n}^{0}$ (b) on spinodal curve and critical solution point of quasiternary systems. Original polymers 1 and 2 , SchulzZimm type distribution $\left(X_{w}{ }^{0}=Y_{w}{ }^{0}=300\right)$ : a) $Y_{w}{ }^{0} / Y_{n}{ }^{0}=$ 2, b) $X_{w}^{0} / X_{n}^{0}=2 ; \chi_{01}=0.2, \chi_{02}=0.3, \chi_{12}=0.01$.

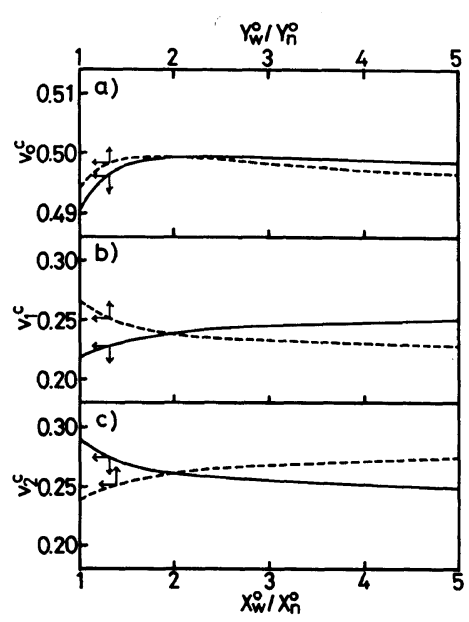

Figure 2. Plots of $v_{0}{ }^{\mathrm{c}}, v_{1}{ }^{\mathrm{c}}$, and $v_{2}{ }^{\mathrm{c}}$ versus $X_{w}{ }^{0} / X_{n}{ }^{\mathrm{o}}$ (full line) or $Y_{w}{ }^{0} / Y_{n}{ }^{0}$ (broken line) of the original polymers 1 and 2 of quasi-ternary systems. Original polymers 1 and 2, Schulz-Zimm type distribution $\left(X_{w^{0}}{ }^{0}=Y_{w^{0}}{ }^{0}=300\right.$; full line, $Y_{w^{0}}{ }^{0} / Y_{n}{ }^{0}=2$; broken line, $\left.X_{w^{\prime}}{ }^{0} / X_{n}{ }^{0}=2\right): \chi_{01}=0.2$, $\chi_{02}=0.3, \chi_{12}=0.01$.

this case $X_{w}{ }^{0} / X_{n}^{0}=Y_{w}{ }^{0} / Y_{n}^{0}=2$ ).

Figure 2 shows the effect of polymer polymolecularity on the composition at the CSP. 

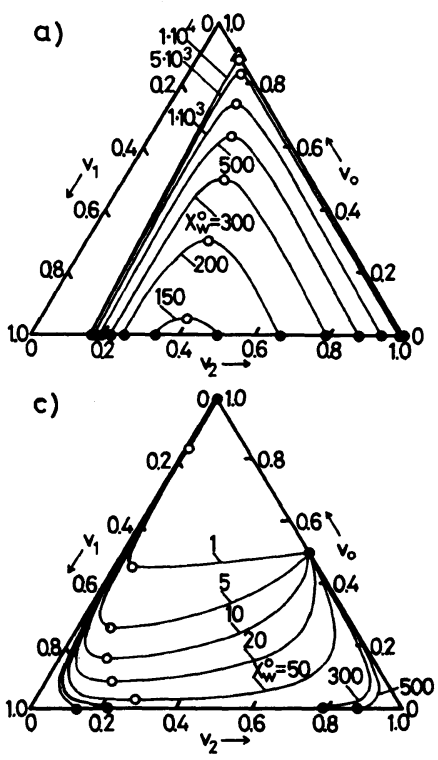
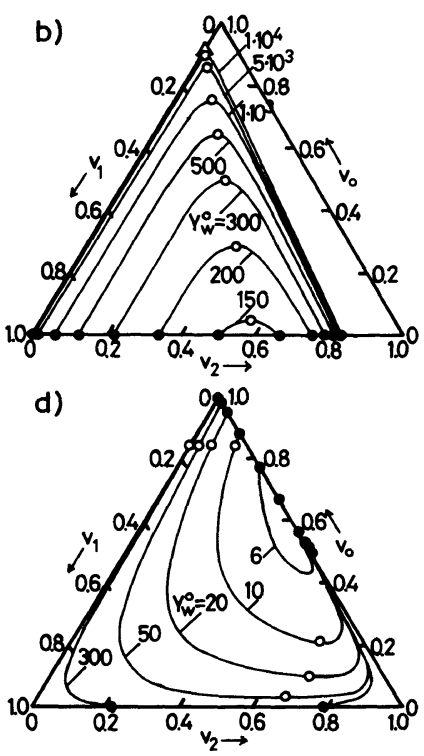

Figure 3. Effect of the average molecular weight of polymer $1\left(X_{w}{ }^{0}, \mathrm{a}\right)$ and c)) and polymer $2\left(Y_{w}{ }^{0}, \mathrm{~b}\right)$ and d)) on spinodal curve and critical solution point of quasi-ternary systems. Original polymers 1 and 2 , Schulz-Zimm type distribution $\left(X_{w}{ }^{0} / X_{n}{ }^{0}=Y_{w}{ }^{0} / Y_{n}{ }^{0}=2\right.$; a) and c) $Y_{w^{0}}{ }^{0}=300$, b) and d) $\left.X_{w}{ }^{0}=300\right): \chi_{01}=0.2$, $\chi_{12}=0.01$, a) and b) $\chi_{02}=0.3$, c) and d) $\chi_{02}=1.0: \bigcirc$, critical solution point; $O$, intersection point of spinodal curve and composition triangle; $\triangle$, Flory solution composition.

As $X_{w}{ }^{0} / X_{n}{ }^{0}$ (full line) or $Y_{w}{ }^{0} / Y_{n}{ }^{0}$ (broken line) increases, $v_{0}{ }^{c}$ increases rapidly, then decreases slowly after passing through maximum. $v_{1}{ }^{c}$ (or $v_{2}^{c}$ ) increases with an increase in $X_{w}{ }^{0} / X_{n}{ }^{0}$ (or $\left.Y_{w}{ }^{0} / Y_{n}{ }^{0}\right)$ and decreases gradually with an increase in $Y_{w}{ }^{0} / Y_{n}{ }^{0}$ (or $X_{w}{ }^{0} / X_{n}{ }^{0}$ ). It is of interest that the effect of polymolecularity becomes remarkable when the range $X_{w}{ }^{0} / X_{n}{ }^{0}$ (or $Y_{w}{ }^{0} /$ $\left.X_{n}{ }^{0}\right)$ is less than 2 .

Figure 3 shows the effects of the weightaverage degrees of polymerization, $X_{w}{ }^{0}$ and $Y_{w}{ }^{0}$, on SC (full line) and CSP (open circle). Here, $\chi_{01}$ and $\chi_{12}$ are taken as 0.2 and 0.01 , respectively and $\chi_{02}$ is taken as 0.3 (Figure $3 \mathrm{a}$ and $b$ ) or 1.0 (Figure $3 \mathrm{c}$ and $\mathrm{d}$ ). The polymers 1 and 2 are assumed to have the Schulz-Zimm type MWD $\left(X_{w}{ }^{0} / X_{n}{ }^{0}=Y_{w}{ }^{0} / Y_{n}{ }^{0}=2\right.$, and $Y_{w}{ }^{0}=$ 300 in Figure $3 \mathrm{a}$ and $3 \mathrm{c}$ and $X_{w}{ }^{0}=300$ in Figure $3 \mathrm{~b}$ and $3 \mathrm{~d}$ ). At $\chi_{02}=0.3$ with an increase in $X_{w}{ }^{0}$ (Figure 3a) the area of single phase region decreases and CSP approaches a single point on the $v_{0}$ axis (as shown as unfilled triangle) which corresponds to the Flory sol- vent composition of the multicomponent polymer/binary solvent mixture system. ${ }^{21} \mathrm{We}$ denote this point as the "Flory (or $\theta$ ) solution composition." The composition of this point $\left(v_{0}{ }^{F}, v_{1}{ }^{F}, v_{2}{ }^{\boldsymbol{F}}\right)$ is given by

$$
\begin{aligned}
&\left(\frac{1}{v_{0}}-2 \chi_{01}\right)\left(\frac{1}{v_{0}}+\frac{1}{v_{2} Y_{w}{ }^{0}}-2 \chi_{02}\right) \\
&-\left(\frac{1}{v_{0}}+\chi_{12}-\chi_{01}-\chi_{02}\right)^{2}=0
\end{aligned}
$$

and in this case $\left(v_{0}^{F}, v_{1}^{F}, v_{2}^{F}\right)=(0.8934,0$, 0.1066). Similalry, with an increase in $Y_{{ }^{*}}{ }^{0}$ (Figure $3 b, \chi_{02}=0.3$ ) CSP approaches the "Flory solution composition" (unfilled triangle) which is evaluated by eq 25

$$
\begin{aligned}
&\left(\frac{1}{v_{0}}+\right.\left.\frac{1}{v_{1} X_{w}^{0}}-2 \chi_{01}\right)\left(\frac{1}{v_{0}}-2 \chi_{02}\right) \\
&-\left(\frac{1}{v_{0}}+\chi_{12}-\chi_{01}-\chi_{02}\right)^{2}=0
\end{aligned}
$$

and in this case $\left(v_{0}{ }^{F}, v_{1}{ }^{F}, v_{2}{ }^{F}\right)=(0.9263,0.0737$, 


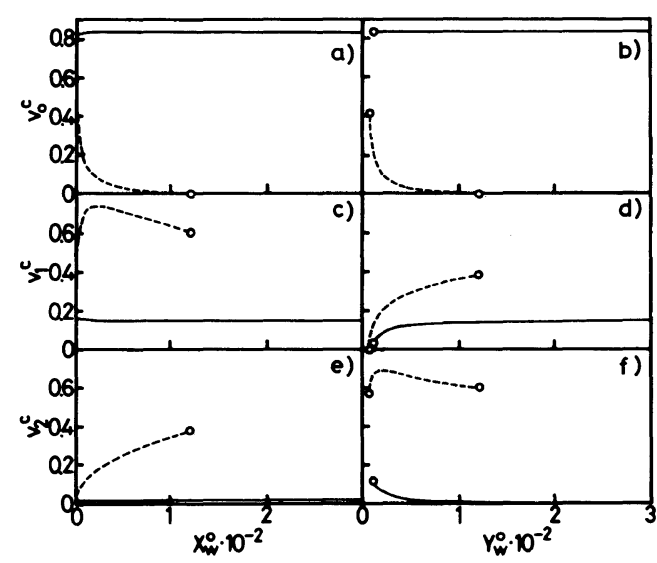

Figure 4. Plots of $v_{0}{ }^{\mathrm{c}}, v_{1}{ }^{\mathrm{c}}$, and $v_{2}{ }^{\mathrm{c}}$ against $X_{w^{0}}{ }^{0}$ (a, c, and d) or $Y_{w}{ }^{0}(\mathrm{~b}, \mathrm{~d}$, and $\mathrm{f})$ of quasi-ternary solutions. Original polymers 1 and 2, Schulz-Zimm type distribution $\left(X_{w}{ }^{0} / X_{n}{ }^{0}=Y_{w}{ }^{0} / Y_{n}{ }^{0}=2 ; \mathrm{a}, \mathrm{c}\right.$, and e, $Y_{w^{0}}{ }^{0}=300 ; \mathrm{b}$, $\mathrm{d}$, and $\left.\mathrm{f}, X_{w^{\prime}}{ }^{0}=300\right): \chi_{01}=0.2, \chi_{02}=1.0, \chi_{12}=0.01$ : full line, critical solution point in higher $v_{0}$ region; broken line, critical solution point in lower $v_{0}$ region: $\bigcirc$, limiting composition of CSP within phase triangle.

0 ). Equations 24 and 25 can be readily derived from the combination of eq 21 and 22 (see Appendix C). ${ }^{21}$ When $\chi_{02}=1.0$, with decrease in $X_{w}{ }^{0}$ (Figure $3 \mathrm{c}$ ) the SC approaches to that for a polymer/binary solvent mixture, and with a decrease in $Y_{w}{ }^{0}$ (Figure 3d) an interesting phenomenon, that although mutual solubility exists between polymer 1 and solvent and between polymers 1 and 2 , the solution of solvent and polymer 2 does not dissolve polymer 1. This phenomenon is similar to cononsolvency, already observed in the computer experiments by Matsuda and Kamide. ${ }^{22}$ The filled circle in Figure $3 a-d$ is the point of intersection between composition triangle and spinodal curve (see, eq 26-28).

Figure 4 illustrates $X_{w}{ }^{0}$ (or $Y_{w}{ }^{0}$ ) dependence of $v_{0}{ }^{c}, v_{1}{ }^{c}$, and $v_{2}{ }^{c}$ at $\left(\chi_{01}, \chi_{02}, \chi_{12}\right)=(0.2,1.0$, 0.01) (namely, $\chi_{01} \neq \chi_{02}$ ). Evidently, the systems have two CSP in the range of $X_{w}{ }^{0}=$ $1 \sim 120$ for a fixed $Y_{w}{ }^{0}(=300)$ and $Y_{w}{ }^{0}=$ $10 \sim 120$ for fixed $X_{w}{ }^{0}(=300)$ (see, Figure $3 \mathrm{c}$ and $\mathrm{d}) . v_{0}^{c}, v_{1}^{c}$, and $v_{2}^{c}$ found in higher $v_{0}$ region are almost constant over a wide range of $X_{w}{ }^{0}$ and $Y_{w}{ }^{0}$ (Figure $4 \mathrm{a}-\mathrm{f}$ ). But in rela-
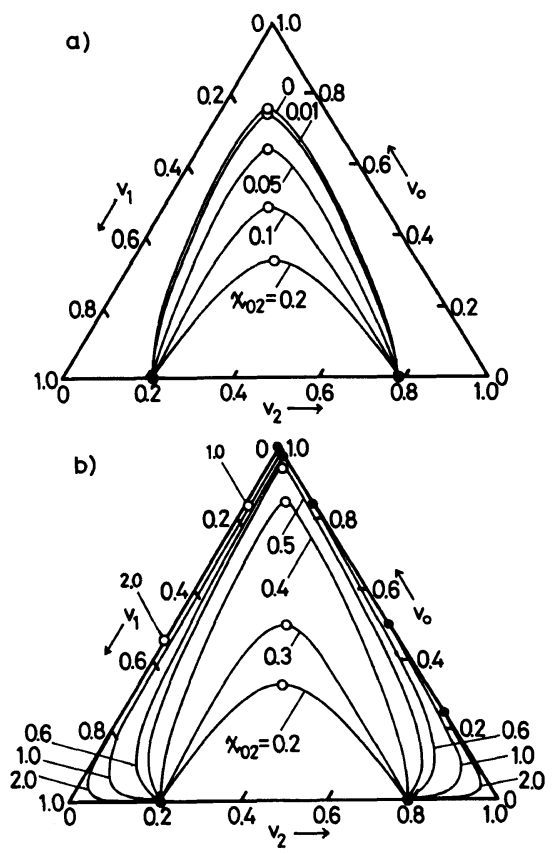

Figure 5. Effect of $\chi_{02}$ on spinodal curve and CSP of quasi-ternary system consisting of multicomponent polymer 1 , multicomponent polymer 2 , and single solvent. Original polymers 1 and 2, Schulz-Zimm type distribution $\left(X_{w^{0}}{ }^{0}=Y_{w^{0}}{ }^{0}=300, X_{w^{*}}{ }^{0} / X_{n}{ }^{0}=Y_{w^{*}}{ }^{0} / Y_{n}{ }^{0}=2\right): \chi_{12}=$ $0.01, \chi_{01}=0.2$; a) $\chi_{02}=0-0.2$ and b) $\chi_{01}=0.2-2.0$; $\bigcirc$, critical solution point; $\boldsymbol{O}$, intersection point of spinodal curve and composition triangle.

tively small $Y_{w}{ }^{0}$ region (with $X_{w}{ }^{0}=300$ ), $v_{1}{ }^{c}$ decreases (and $v_{2}{ }^{c}$ increases) gradually with decrease in $Y_{w}{ }^{0}$ and at $Y_{w}{ }^{0} \lesssim 8$ CSP disappears (in other words, CSP moves to the outside of the compoisiton triangle; see Figure $3 \mathrm{~d}$ ). The open circles at the end of solid line in Figure $4 \mathrm{~b}, \mathrm{~d}$, and e show the compositions of CSP at $Y_{w}{ }^{0} \simeq 8$. When $Y_{w}{ }^{0}$ is smaller than 5 (for fixed $\left.X_{w}{ }^{0}(=300)\right)$ polymers 1 and 2 are perfectly miscible throughout the entire composition region. In the lower $v_{0}$ region, $v_{0}{ }^{c}$ decreases with increase in $X_{w^{0}}{ }^{0}$ (or $Y_{w}{ }^{0}$ ) (broken line in Figure $4 \mathrm{a}$ (or b), approaching zero at $X_{w}{ }^{0} \simeq$ 120 (or $Y_{w}{ }^{0} \simeq 120$ ) (open circle in the figures). As $X_{w}{ }^{0}$ increases at a fixed $Y_{w}{ }^{0}(=300), v_{1}{ }^{c}$ increases first and then decreases after passing a minimum but $v_{2}{ }^{c}$ increases monotonously (broken line in Figure $4 c$ and e). 
$Y_{w}{ }^{0}$ dependence of $v_{1}{ }^{c}$ and $v_{2}{ }^{c}$ with a constant $X_{w}{ }^{0}(=300)$ is similar with their $X_{w}{ }^{0}$ dependence at constant $Y_{w}{ }^{0}$, but the details are different.

Figure $5 \mathrm{a}$ and $\mathrm{b}$ show the effect of $\chi_{02}$ on the SC (full line) and the CSP (open circle). Here $\chi_{01}=0.2$ and $\chi_{12}=0.01$. Polymers 1 and 2 are assumed to have the Schulz-Zimm type distribution with $X_{w}{ }^{0}=Y_{w}{ }^{0}=300$ and $X_{w}{ }^{0} /$ $X_{n}{ }^{0}=Y_{w}{ }^{0} / Y_{n}^{0}=2$. At $\chi_{02}=0.2\left(=\chi_{01}\right.$, in this case) area of single phase region becomes maximum. In other words, usage of single solvent having similar solubility against polymer 1 and polymer 2 widens effectively the single phase region. When $\chi_{02}$ ranges from 0.6 to 0.8 , CSP cannot be found within the compositional triangle diagram (i.e., the critical solution point does not exist). In the range $0.8<\chi_{02}<2.0$, the critical solution point lies nearly on the $v_{1}$ axis.

The crossing point of SC and the three axis $\left(v_{0}, v_{1}\right.$, and $v_{2}$ axes) of the triangle diagram (filled circle) can be theoretically calculated according to the following equations, respectively.

$$
\begin{aligned}
& \frac{v_{0}}{Y_{w}{ }^{0}}+v_{2}-2 \chi_{02} v_{0} v_{2}=0 \\
& \frac{v_{0}}{X_{w}{ }^{0}}+v_{1}-2 \chi_{01} v_{0} v_{1}=0 \\
& \frac{v_{1}}{X_{w}{ }^{0}}+\frac{v_{2}}{Y_{w}{ }^{0}}-2 \chi_{12} v_{1} v_{2}=0
\end{aligned}
$$

Equations $26-28$ are readily derived from eq B-8 (see Appendix B). The intersection point of SC and $v_{2}$-axis (polymers $1-2$ axis) are independent of $\chi_{02}$ and are determined by the combination of $X_{w}{ }^{0}, Y_{w}{ }^{0}$, and $\chi_{12}$ (see, eq 28). In this case $\left(v_{0}, v_{1}, v_{2}\right)=(0,0.2113,0.7887)$ and $(0,0.7887,0.2113)$.

Figure 6 shows the effect of $\chi_{02}$ on $v_{0}^{c}$, $v_{1}^{c}$, and $v_{2}^{c}$. At $\chi_{02}\left(=\chi_{01}\right)=0.2, v_{0}^{c}$ attains a minimum and both $v_{1}{ }^{c}$ and $v_{2}{ }^{c}$ reach a maximum. In the range $0.55 \lesssim \chi_{02} \lesssim 0.82$, the spinodal curve intersects with the $v_{0}$-axis (solvent-polymer 2 axis) and CSP moves to

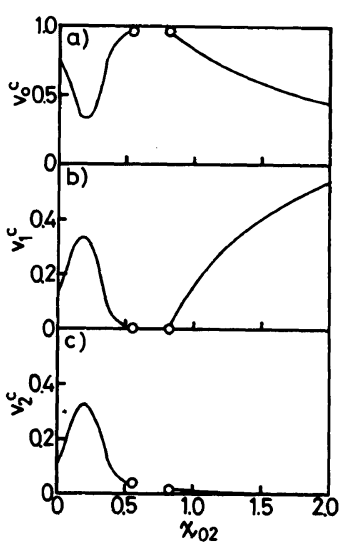

Figure 6. Dependence of the composition of CSP, $v_{0}{ }^{\mathbf{c}}$, $v_{1}{ }^{\mathrm{c}}$, and $v_{2}{ }^{\mathrm{c}}$ on $\chi_{02}$ of quasi-ternary systems. Original polymers 1 and 2, Schulz-Zimm type distribution $\left(X_{w^{0}}{ }^{0}=\right.$ $\left.300, X_{w}{ }^{0} / X_{n}{ }^{0}=2, Y_{w}{ }^{0}=300, Y_{w}{ }^{0} / Y_{n}{ }^{0}=2\right)$ : full line, $\chi_{01}=$ 0.2 ; broken line, $\chi_{01}=1.0$.

outside of composition triangle (see, Figure $5 b)$. The intersection point can be calculated by use of eq 26. In the region of $\chi_{02} \gtrsim 0.82$. CSP appears again near $v_{1}$-axis (solvent-polymer 2 axis) and $v_{1}^{c}$ increases and $v_{0}{ }^{c}$ decreases the increase in $\chi_{02}$ (see, Figure $5 \mathrm{~b}$ and $6 \mathrm{~b}$ ).

Figure $7 \mathrm{a}-\mathrm{d}$ show the effect of $\chi_{12}$ on SC (full line) and CSP (open circle). Here $\chi_{01}$ is taken as constant $(0.2)$ and $X_{w}{ }^{0}=Y_{w}{ }^{0}=300$ and $X_{w}{ }^{0} / X_{n}{ }^{0}=Y_{w}{ }^{0} / Y_{n}{ }^{0}=2$. In the case of $\chi_{02}=$ 0.3 (Figure $7 \mathrm{a}$ ) polymers 1 and 2 are almost incompatible at $\chi_{12}=0.1$ and accordingly there is a very narrow one phase region whose area increases with decrease in $\chi_{12}$. By use of eq 28 , we can conclude that no two phase region exists when $\chi_{12}<1 / 150(\simeq 0.0067)$. When $v_{0}$ reduces zero, the system approaches the multicomponent polymers 1/multicomponent polymers 2 system (i.e., quasi-binary polymer blend), of which thermodynamics will be discussed in detail in the forthcoming paper. ${ }^{23}$ At $\chi_{12}=0.4$ (Figure $7 \mathrm{~b}$ ) SCs are "closed loops" and two CSPs are observed when $\chi_{12}<1 / 150\left(\chi_{12}=0\right.$ and 0.004 in the figure), as were calculated by Zemann and Patterson. ${ }^{11}$ This phenomenon may be related to the cononsolvency. ${ }^{18}$

At $\chi_{02}=0.6$ and 0.8 (Figure $7 \mathrm{c}$ and $\mathrm{d}$ ), SCs 

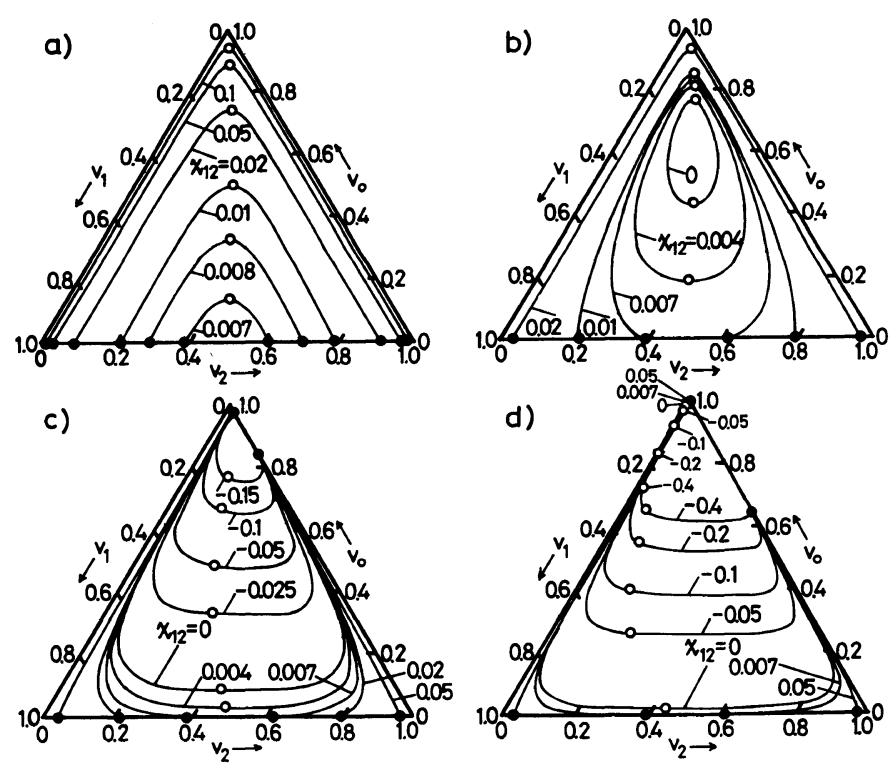

Figure 7. Effects of $\chi_{12}$ on spinodal curve and CSP of quasi-ternary systems. Original polymers 1 and 2 , Schulz-Zimm type distribution $\left(X_{w}{ }^{0}=Y_{w}{ }^{0}=300, X_{w}{ }^{0} / X_{n}{ }^{0}=Y_{w}{ }^{0} / Y_{n}{ }^{0}=2\right) ; \chi_{01}=0.2$, a) $\chi_{02}=0.3$, b) $\chi_{02}=$ 0.4 , c) $\chi_{02}=0.6$, and d) $\chi_{02}=0.8: \bigcirc$, critical solution point; $O$, intersection points of spinodal curve and axes of composition triangle graph.

interesect with $v_{0}$-axis (solvent-polymer 2 axis) and CSP at higher $v_{0}$ side diminishes. Intersection points are evaluated as $\left(v_{0}, v_{1}\right.$, $\left.v_{2}\right)=(0.8489,0,0.1511)$ and $(0.9816,0$, $0.0184)$ for $\chi_{02}=0.6$ and $\left(v_{0}, v_{1}, v_{2}\right)=(0.6285$, $0,0.3715)$ and $(0.9944,0,0.0056)$ for $\chi_{02}=0.8$, by eq 26 . In the case of $\chi_{02}=0.8$, another CSP are observed near $v_{1}$ axis in higher $v_{0}$ region and two CSPs approach as $\chi_{12}$ decreases.

Summarizing, the equations of spinodal and neutral equilibrium conditions (eq 21 and 22, respectively) were derived for multicomponent polymers 1 /multicomponent polymers 2 /single solvent systems and using these equations, SC can be calculated from $\chi_{01}, \chi_{02}, \chi_{12}, X_{w}{ }^{0}$, and $Y_{w}{ }^{0}$ and CSP can be evaluated from $\chi_{01}, \chi_{02}$, $\chi_{12}, X_{w}{ }^{0}, Y_{w}{ }^{0}, X_{z}{ }^{0}$, and $Y_{z}{ }^{0}$. With increase in the breadth of the MWD of a given polymer
CSP shifted in the triangle phase diagram to the side of the polymer. The critical solvent volume fraction $v_{0}^{c}$ gets large with increase in either $X_{w}{ }^{0}$ or $Y_{w}{ }^{0}$. In particular, at the limit of $X_{w}{ }^{0} \rightarrow \infty$ ( or $Y_{w}{ }^{0} \rightarrow \infty$ ) CSP approaches "Flory solvent composition," which was theoretically predicted (eq 24 or 25). Equations giving the cross points of the polymer 1-polymer 2 axis of the triangle diagram and SC were also derived. Solving these equations, the conditions of appearance of closed loop type two-phase region were determined.

\section{APPENDIX A}

Derivation of eq $16^{\prime}$ and 18':

Substituting eq $15 \mathrm{a}-\mathrm{e}$ into eq 10 , eq 16 is obtained: 
Phase Equilibria of Quasi-Ternary Systems I

$$
\left|\Delta G^{\prime}\right|=\left(\frac{R T}{V_{0}}\right)^{m_{1}+m_{2}}\left|\begin{array}{ccccccccc}
M+M_{1} & M & \cdots & M & \vdots & K & K & \cdots & K \\
M & M+M_{2} & \ldots & M & \vdots & K & K & \cdots & K \\
\vdots & \vdots & & \vdots & \vdots & \vdots & \vdots & & \vdots \\
M & M & & M+M_{m_{1}} & \vdots & K & K & \ldots & K \\
\ldots & \ldots & \ldots & \ldots & \ldots & \ldots & \ldots & \ldots & \ldots \\
K & K & \ldots & K & \vdots & N+N_{1} & N & \ldots & N \\
K & K & \ldots & K & \vdots & N & N+N_{2} & \cdots & N \\
\vdots & \vdots & & \vdots & \vdots & \vdots & \vdots & & \vdots \\
K & K & \ldots & K & \vdots & N & N & \cdots & N+N_{m_{2}}
\end{array}\right|
$$

By use of the addition rule, Laplace expansion, and cofactor expansion of determinant, $\left|\Delta G^{\prime}\right|$ becomes

$$
\begin{aligned}
& \left(\frac{V_{0}}{R T}\right)^{m_{1}+m_{2}}\left|\Delta G^{\prime}\right|=\left|\begin{array}{ccc}
M-K+M_{1} & \cdots & M-K \\
\vdots & \ddots & \vdots \\
N-K & \cdots & M-K+M_{m_{1}}
\end{array}\right| \times\left|\begin{array}{ccc}
N+N_{1} & \ldots & N \\
\vdots & \vdots & \vdots \\
N & \cdots & N+N_{m_{2}}
\end{array}\right|
\end{aligned}
$$

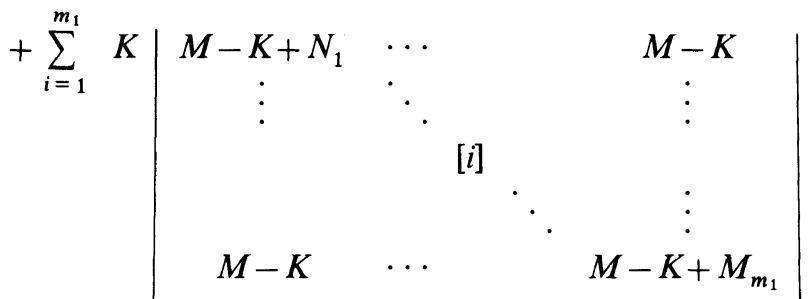

$$
\begin{aligned}
& \left.-\sum_{j \neq i}^{m_{i}} K(M-K) \frac{1}{M_{i} M_{j}} \prod_{k=1}^{m_{1}} M_{k}\right] \times\left|\begin{array}{ccc}
N-K+N_{1} & \cdots & N-K \\
\vdots & \ddots & \vdots \\
N-K & \cdots & N-K+N_{m_{1}}
\end{array}\right| \\
& =\left(\prod_{k=1}^{m_{1}} M_{k}\right)\left[1+(M-K) \sum_{k=1}^{m_{1}} \frac{1}{M_{k}}\right] \times\left(\prod_{k=1}^{m_{2}} N_{k}\right) \times\left[1+N \sum_{k=1}^{m_{2}} \frac{1}{N_{k}}\right] \\
& +\sum_{i=1}^{m_{1}}\left[K \cdot \frac{1}{M_{i}}\left(\prod_{k=1}^{m_{1}} M_{k}\right)\left[1+(M-K)\left\{\sum_{k=1}^{m_{1}} \frac{1}{M_{k}}-\frac{1}{M_{i}}\right\}\right]\right. \\
& \left.-K(M-K) \sum_{j \neq i}^{m_{1}} \frac{1}{M_{i} M_{j}}\left(\prod_{k=1}^{m_{1}} M_{k}\right)\right] \times\left(\prod_{K=1}^{m_{2}} N_{k}\right)\left[1+(N-K) \sum_{k=1}^{m_{2}} \frac{1}{N_{k}}\right]
\end{aligned}
$$

In the derivation of eq $\mathrm{A}-2$, the following relation is used. ${ }^{19}$ 


$$
|D|=\left|\begin{array}{cccc}
x_{1} & a & \cdots & a \\
a & x_{2} & & \vdots \\
\vdots & & \ddots & \\
a & \cdots & & x_{n}
\end{array}\right|=f(a)-a \frac{\mathrm{d} f(x)}{\mathrm{d} x}
$$

where

and

$$
|D|=\prod_{i=1}^{m}\left(x_{i}-a\right)\left[1+a \sum_{k=1}^{m_{1}} \frac{1}{x_{k}-a}\right]
$$

Now, we can readily rewrite eq A-2 as eq $16^{\prime}$.

Equation 18 can be rewritten as eq A-6 with the help of a cofactor expansion, Laplace expansion, and eq $16^{\prime}$.

$$
\begin{gathered}
f(x)=\prod_{i=1}^{m}\left(x_{i}-x\right) \quad(\mathrm{A}-4) \\
\left(\frac{V_{0}}{R T}\right)^{m_{1}+m_{2}-1}\left|\Delta G^{\prime \prime}\right|=W_{X_{1}}\left(\frac{1}{M_{k=1}} \prod_{k=1}^{m_{1}} M_{k}\right)\left(\prod_{k=1}^{m_{2}} N_{k}\right)\left[\left\{1+M\left(\sum_{k=1}^{m_{1}} \frac{1}{M_{k}}-\frac{1}{M_{1}}\right)\right\}\left(1+N \sum_{k=1}^{m_{2}} \frac{1}{N_{k}}\right)\right. \\
\left.-K^{2}\left(\sum_{k=1}^{m_{1}} \frac{1}{M_{k}}-\frac{1}{M_{1}}\right)\left(\sum_{k=1}^{m_{2}} \frac{1}{N_{k}}\right)\right]-\sum_{i=2}^{m_{1}} W_{X_{i}}\left[\frac{M}{M_{1} M_{i}}\left(\prod_{k=1}^{m_{1}} M_{k}\right)\left(\prod_{k=1}^{m_{2}} N_{k}\right)\left(1+N \sum_{k=1}^{m_{2}} \frac{1}{N_{k}}\right)\right. \\
\left.-\sum_{j=1}^{m_{2}} \frac{K^{2}}{M_{1} M_{i}}\left(\prod_{k=1}^{m_{1}} M_{k}\right) \frac{1}{N_{j}}\left(\prod_{k=1}^{m_{2}} N_{k}\right)\right]-\sum_{i=1}^{m_{2}} W_{Y_{j}} \frac{K}{M_{1}}\left(\prod_{k=1}^{m_{1}} M_{k}\right) \frac{1}{N_{i}}\left(\prod_{k=1}^{m_{2}} N_{k}\right) \\
=\frac{1}{M_{1}}\left(\prod_{k=1}^{m_{1}} M_{k}\right)\left(\prod_{k=1}^{m_{2}} N_{k}\right)\left[\left(\sum_{i=1}^{m_{1}} \frac{W_{X_{i}}}{M_{i}}\right)\left\{K^{2} \sum_{j=1}^{m_{2}} \frac{1}{N_{j}}-M\left(1+M \sum_{j=1}^{m_{2}} \frac{1}{N_{j}}\right)\right\}-K\left(\sum_{j=1}^{m_{2}} \frac{W_{Y_{j}}}{N_{j}}\right)\right]
\end{gathered}
$$

Combination of eq A-6 and $16^{\prime}$ yields $18^{\prime}$.

\section{APPENDIX B}

Symmetry of eq 21 and 22

By introducing parameters $Q_{X}$ and $Q_{Y}$ defined by following equations,

$$
\begin{aligned}
\frac{1}{Q_{X}} & \equiv \sum_{i=1}^{m_{1}} \frac{1}{M_{i}}=v_{1} X_{w}{ }^{0} \\
\frac{1}{Q_{Y}} & \equiv \sum_{j=1}^{m_{2}} \frac{1}{N_{j}}=v_{2} Y_{w}{ }^{0}
\end{aligned}
$$

into eq 16 we obtain

$$
\left|\begin{array}{cc}
M+Q_{X} & K \\
K & M+Q_{Y}
\end{array}\right|=0
$$

Equation B-2 can be rewritten as:

$$
\begin{aligned}
\left(M+Q_{X}-K\right)(N & \left.+Q_{Y}-K\right)+K\left(M+Q_{X}-K\right) \\
& +K\left(N+Q_{Y}-K\right)=0
\end{aligned}
$$

Substitution of eq $15 \mathrm{a}-\mathrm{e}, \mathrm{B}-1 \mathrm{a}$ and $\mathrm{b}$ into eq B-3 yields eq $21^{\prime}$.

The neutral equilibrium condition eq 20 can also be represented as

$$
\left|\begin{array}{cc}
Q_{X} R_{X} & Q_{X} R_{Y} \\
K & N+Q_{Y}
\end{array}\right|=0
$$

where $R_{X}$ and $R_{Y}$ are defined by:

$$
\begin{aligned}
& R_{X} \equiv \sum_{i=1}^{m_{1}} \frac{W_{X_{i}}}{M_{i}} \\
& R_{Y} \equiv \sum_{j=1}^{m_{2}} \frac{W_{Y_{j}}}{M_{j}}
\end{aligned}
$$

Equation B-4 is rewritten as eq B-7 with the help of eq B-3,

$$
\begin{aligned}
\frac{1}{v_{0}^{2}}(M & \left.+Q_{X}-K\right)^{3}\left(N+Q_{Y}-K\right)^{3} \\
& +X_{z}^{0} Q_{X}^{2} K^{3}\left(N+Q_{Y}-K\right)^{3} \\
& +Y_{z}^{0} Q_{Y}^{2} K^{3}\left(M+Q_{X}-K\right)^{3}=0
\end{aligned}
$$


Substituting eq $15 \mathrm{a}-\mathrm{e}, \mathrm{B}-\mathrm{la}$, and $\mathrm{b}$ into eq B7 , eq $22^{\prime}$ is finally obtained. Equation $21^{\prime}$ and $22^{\prime}$ are obviously symmetric with respect to the exchange of polymers 1 and 2, respectively.

Multiplying both sides of eq $21^{\prime}$ by $v_{0} v_{1} v_{2}$ gives

$$
\begin{aligned}
& v_{0}\left\{\frac{1}{X_{w}{ }^{0}}+\left(\chi_{02}-\chi_{01}-\chi_{12}\right)\right\} \\
& \quad \times\left\{\frac{1}{Y_{w}^{0}}+\left(\chi_{01}-\chi_{02}-\chi_{12}\right) v_{2}\right\} \\
& +v_{1}\left\{1+\left(\chi_{12}-\chi_{01}-\chi_{02}\right) v_{0}\right\} \\
& \quad \times\left\{\frac{1}{Y_{w}^{0}}+\left(\chi_{01}-\chi_{02}-\chi_{12}\right) v_{2}\right\} \\
& +v_{2}\left\{1+\left(\chi_{12}-\chi_{01}-\chi_{02}\right) v_{0}\right\} \\
& \quad \times\left\{\frac{1}{X_{w}^{0}}+\left(\chi_{02}-\chi_{01}-\chi_{12}\right) v_{1}\right\}=0
\end{aligned}
$$

By putting $v_{0}=0$ (and of course utilizing $v_{1}+v_{2}=1$ ) in eq B-8, we can obtain eq 28 . Equations 26 and 27 are similarly derived from eq B-8.

\section{APPENDIX C}

\section{Derivation of eq 24 and 25}

Regardless of the MWD of the original polymer, at the limit of $X_{w}{ }^{0} \rightarrow \infty$ (and also $X_{z}^{0} \rightarrow \infty$ ) following relation holds:

$$
\left(v_{0}{ }^{c}, v_{1}{ }^{c}, v_{2}{ }^{c}\right)=\left(v_{0}{ }^{F}, 0, v_{2}{ }^{F}\right)
$$

Assuming $v_{1}{ }^{c}$ approachs zero $\left(=v_{1}{ }^{F}\right)$ with order of $\left(X_{w^{*}}\right)^{-a}(0<a<1)$ with increasing $X_{w^{0}}{ }^{0}$; namely, $1 /\left(v_{1}{ }^{c} X_{w}{ }^{0}\right)\left(\sim O\left(\left(X_{w}{ }^{0}\right)^{a-1}\right)\right) \rightarrow 0$ when $X_{w}{ }^{0} \rightarrow \infty$, we can obtain the equation of "Flory (or $\theta$ ) solution composition" as eq 24 from the equation of spinodal condition (eq 21). As the "Flory solution point" on solvent-polymer 2 axis is the CSP at the limit of $X_{u^{0}}{ }^{0} \rightarrow \infty$, the neutral equilibrium condition (eq 22) is simultaneously satisfied regardless of $v_{0}$ and $v_{2}{ }^{17}$
Equation 25 is also derived in the same manner.

Appendixes A and B are abbreviated at the request of referees. Details of the calculations can be obtained from the authors.

\section{REFERENCES}

1. See, for example, "Polymer Compatibility and Imcompatibility," K. Šolc, Ed., Harwood Academic Publishers, New York, 1982.

2. S. Saeki, Y. Narita, M. Tsubokawa, and T. Yamaguchi, Polymer, 24, 1631 (1983).

3. S. Saeki, Y. Aoyanagi, M. Tsubokawa, and T. Yamaguchi, Polymer, 25, 1779 (1984).

4. T. Fukuda, M. Nagata, and H. Inagaki, Macromolecules, 17, 548 (1984).

5. A. C. Su and J. R. Fried, Macromolecules, 19, 1417 (1986).

6. L. O. Kaddour and Cl. Strazielle, Polymer, 28, 459 (1987).

7. Z. Tong, Y. Einaga, H. Miyashita, and H. Fujita, Macromolecules, 20, 1883 (1987).

8. Z. Tong, Y. Einaga, H. Miyashita, and H. Fujita, Macromolecules, 20, 1888 (1987).

9. Z. Tong, Y. Einaga, and H. Fujita, Polym. J., 19, 965 (1987).

10. R. L. Scott, J. Chem. Phys., 17, 268 (1949).

11. H. Tompa, Trans. Faraday Soc., 45, 1142 (1949).

12. H. Tompa, "Polymer Solutions," Butterwords Scientific Publishers, London, 1956.

13. L. Zemann and D. Patterson, Macromolecules, 5, 513 (1972).

14. C. C. Hsu and J. M. Prausnitz, Macromolecules, 7, 320 (1974).

15. K. Solc, Macromolecules, 19, 1166 (1986).

16. K. Solc, Macromolecules, 20, 2506 (1987).

17. R. Koningsveld, H. A. G. Chermin, and M. Gordon, Proc. R. Soc. London Ser. A, 319, 331 (1970).

18. R. Koningsveld, Discuss. Faraday Soc., 49, 144 (1970).

19. K. Kamide and S. Matsuda, Polym. J., 18, 347 (1986).

20. P. J. Flory, "Principle of Polymer Chemistry," Cornell Univ. Press, Ithaca, 1953.

21. S. Matsuda and K. Kamide, Polym. J., 19, 203 (1987).

22. S. Matsuda and K. Kamide, Polym. J., 19, 211 (1987)

23. K. Kamide, H. Shirataki, and S. Matsuda, submitted to Polym. J. 